\title{
A Theoretical Study of Electronic and Vibrational Properties of Neutral, Cationic, and Anionic $\mathrm{B}_{24}$ Clusters
}

\author{
KAH CHUN LAU, MRINALINI DESHPANDE, ${ }^{*}$ RANJIT PATI, \\ RAVINDRA PANDEY
}

Michigan Technological University, Houghton, MI 49931

Received 18 November 2004; accepted 5 December 2004

Published online 4 March 2005 in Wiley InterScience (www.interscience.wiley.com).

DOI 10.1002/qua.20537

\begin{abstract}
The equilibrium geometries, electronic and vibrational properties, and static polarizability of $\mathrm{B}_{24}, \mathrm{~B}_{24}^{-}$, and $\mathrm{B}_{24}^{+}$clusters are reported here. First-principles calculations based on density functional theory predict the staggered double-ring configuration to be the ground state for $\mathrm{B}_{24}, \mathrm{~B}_{24}^{-}$, and $\mathrm{B}_{24}^{+}$, in contrast to the quasi-planar structure observed in small neutral and ionized $\mathrm{B}_{n}$ clusters with $n \leq 15$. Furthermore, the $\left(4 \times \mathrm{B}_{6}\right)$ tubular structure is found to be relatively stable in comparison to the 3D cage structure. The presence of delocalized $\pi$ and multicentered $\sigma$ bonds appears to be the cause of the stability of the double-ring and tubular isomers. For the ground state of $\mathrm{B}_{24}$, the lower and upper bound of the electron affinity is 2.67 and $2.81 \mathrm{eV}$, respectively, and the vertical ionization potential is $6.88 \mathrm{eV}$. Analysis of the frequency spectrum of the double-ring and tubular isomers reveals the characteristic vibrational modes typically observed in carbon nanotubes. The corresponding IR spectrum also reflects the presence of some of these characteristic modes in the neutral and ionized $\mathrm{B}_{24}$, suggesting that double-ring and tubular structures can be considered as the building blocks of boron nanotubes. (C) 2005 Wiley Periodicals, Inc. Int J Quantum Chem 103: $866-874,2005$
\end{abstract}

Key words: nanoclusters; boron; vibrational properties

\section{Introduction}

$\mathbf{F}$ ollowing the discovery of carbon fullerenes [1] and carbon nanotubes [2], interest in exploring the novel nanostructures has opened a new

Correspondence to: R. Pandey; e-mail: pandey@mtu.edu

*Permanent Address: Department of Physics, H.P.T. Arts and R.Y.K. Science College, Nasik, India. horizon in the field of materials science. In this, boron holds the unique place by having the most varied polymorphism, which includes quasi-crystal [3], nanowire [4], nanoribbon [5], and nanotube [6]. Besides the interesting physical and chemical properties of small boron clusters, it is observed that the structural arrangement of $\mathrm{B}_{n}$ clusters [7-13] $(n \leq 15)$ is very different from its bulk structure [14-17]. The small elemental boron clusters have either quasiplanar or convex structure [7-13] instead of the 
highly symmetric icosahedral structure, observed in the conventional boron-rich solids [14-17]. The experimental results on the synthesis of single-wall boron nanotubes report that boron nanotubes are extremely sensitive to the high-energy electron exposure. As a result, both length and structure of the nanotubes were changed significantly and the nanotubes were ultimately destroyed [6]. Thus, before understanding stability and electronic properties of a large tubular structure of boron, it is necessary to understand the physics and chemistry of large boron clusters.

Most of the previous studies have considered only the medium-size boron clusters, calculating their structural and electronic properties in the neutral charge state $[18,19]$. Calculations of the vibrational properties and infrared spectra were not performed. It is well known that calculations of the vibrational frequencies of a given cluster can only determine its configurational stability. In our research group, we have initiated such a study of neutral and ionized $\mathrm{B}_{n}$ clusters, determining their configurational stability by analyzing their vibrational spectra. The results for our calculations for the neutral $\mathrm{B}_{12}$ cluster show that the most stable configuration is a convex structure with $C_{1}$ symmetry, in contrast to the previously reported $\mathrm{C}_{3 v}$ structure $[18,20]$. The calculated results also find the ionized $\mathrm{B}_{12}^{+}$to be less stable than the neutral $\mathrm{B}_{12}$ [20].

As a next step, we report the results on the configurational stability and vibrational properties of the neutral and ionized $B_{24}$ in this article. We note here that equilibrium geometries of $B_{24}$ were reported previously $[18,19]$ in which a symmetryconstrained optimization [18] found the doublering configuration to be the lowest energy configuration for the neutral $B_{24}$. A recent density functional theory (DFT) study considering several isomers including ring, convex, quasi-planar, tubular, and closed structures has confirmed the doublering isomer to be the ground state of $B_{24}$ [19]. However, none of the previous studies have investigated the configuration stability of $B_{24}$ in terms of the vibrational frequency. Furthermore, we are not aware of any theoretical study on the ionized $\mathrm{B}_{24}^{-}$ and $\mathrm{B}_{24}^{+}$clusters, which are important in determining the ionization-induced distortion together with the values of ionization potential and electron affinity of $B_{24}$.

This article is organized as follows: In Section 2, we briefly describe the computational details. Results and discussion of geometrical features, cluster stability, electronic properties, chemical bonding, vibrational frequencies, and static dipole polarizability of the neutral and ionized $B_{24}$ are presented in Section 3. A summary of the calculated results is given in Section 4.

\section{Computational Method}

Electronic structure calculations were performed on several neutral, anionic, and cationic isomers of $\mathrm{B}_{24}$ using the Gaussian 98 code [21]. All calculations were carried out by solving Kohn-Sham equations in the framework of density functional theory. We employed the generalized gradient approximations (GGA) using the functionals of Becke's three-parameter hybrid exchange functional and Lee-YangParr correlation functional (B3LYP) and a doublezeta basis set (i.e., 6-31G $(d, p)$ or $6-31 G^{* *}$ ) in these calculations. We considered the reliability and accuracy of the $6-31 G(d, p)$ basis set in our previous study of $B_{12}$ [20] and found that the use of a larger basis set, e.g., the 6-311G(df) basis set, does not introduce significant changes in structural energetics and electronic properties of $B_{12}$ obtained by the 6-31G $(\mathrm{d}, \mathrm{p})$ basis set.

All the structures have been fully optimized by employing the gradient and updated Hessian. The convergence criteria for the gradient and energy were set to $10^{-4}$ hartree/ $\AA$ and $10^{-9}$ hartree, respectively. The stability of the isomers considered was addressed by computing the vibrational frequencies under the harmonic approximation with analytical force constants. We have also calculated the static dipole polarizability of all the structures by applying an external electric field of strength 0.001 au along the $x, y$, and $z$ axes separately.

\section{Results and Discussion}

\subsection{STRUCTURES AND ENERGETICS}

All initial configurations considered for $\mathrm{B}_{24}$ in this study are shown in Figure 1. Rather than an exhaustive exploration of the potential energy surfaces of $\mathrm{B}_{24}$, which is very onerous because of the factorially large number of possible isomers involved, we restrict ourselves to isomers that can be classified into the representatives, such as 2D quasiplanar, chain, tubular, and closed 3D structures, based on the previous studies [18, 19, 22]. Given that the monocyclic ring structure of $B_{24}$ is found to be relatively less stable among these isomeric con- 

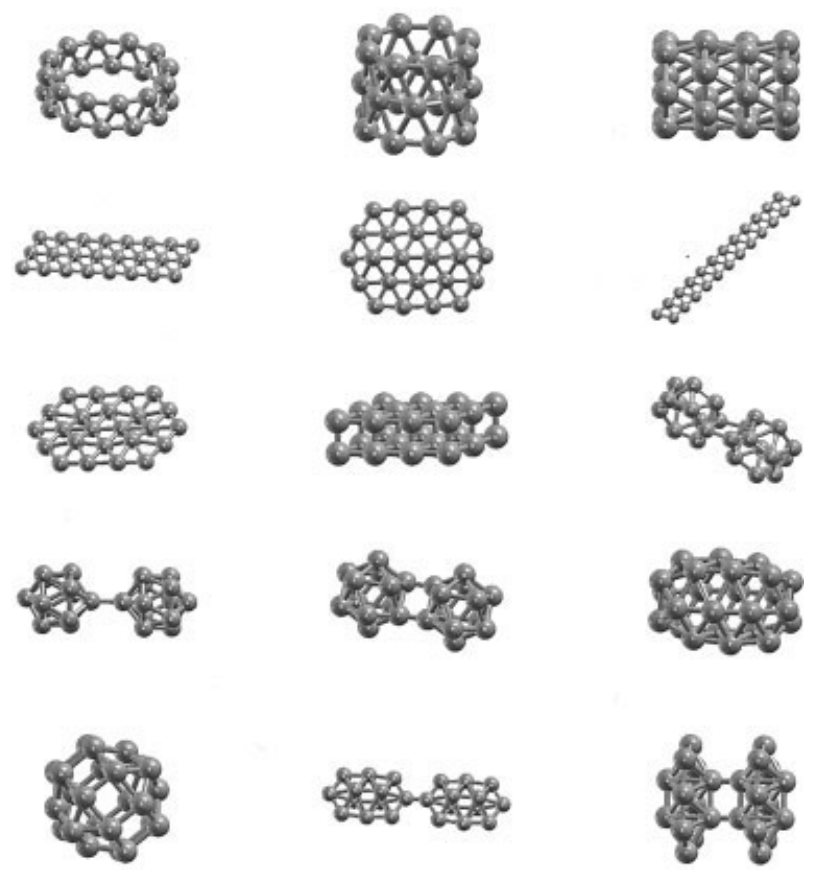

FIGURE 1. Some of the isomers of $B_{24}$ clusters considered in this study.

figurations, we have not considered the monocyclic ring together with the linear chain configurations in the electronic structure calculations. The ordering of the optimized isomeric configurations for selected neutral and charged $\mathrm{B}_{24}$ in terms of the calculated total energy is shown in Figure 2.

\subsubsection{Neutral $B_{24}$}

The lowest energy configuration of the neutral $\mathrm{B}_{24}$ cluster is the double-ring structure $\left(\mathrm{B}_{24}-\mathrm{I}\right)$ with $\mathrm{C}_{4 v}$ symmetry and ${ }^{1} \mathrm{~A}_{1}$ electronic state. Two rings of 12 boron atoms, each of diameter with $6.25 \AA$ and $1.68 \AA$ apart are arranged in a staggered configuration. The coordination number of $\mathrm{B}$-atom in this double-ring structure is 4 and $R_{\mathrm{B}-\mathrm{B}}$ is $1.62 \AA$. Each ring is rotated by an angle of $\pi / 12$ with respect to the other ring. This staggered configuration facilitates the $\mathrm{sp}^{2}$ hybridization between boron atoms, which makes this structure comparatively stable among the tubular structures. The binding energy (BE) of this structure is $5.04 \mathrm{eV} /$ atom as shown in Figure 3. We note that the DFT-planewave calculations [19] report a higher binding energy of 5.95 $\mathrm{eV} /$ atom that very close to the bulk boron of 6.0 $\mathrm{eV} /$ atom [23]. We believe that it may be due to a smaller diameter of $6.22 \AA$ and a smaller $R_{\mathrm{B}-\mathrm{B}}$ of 1.45

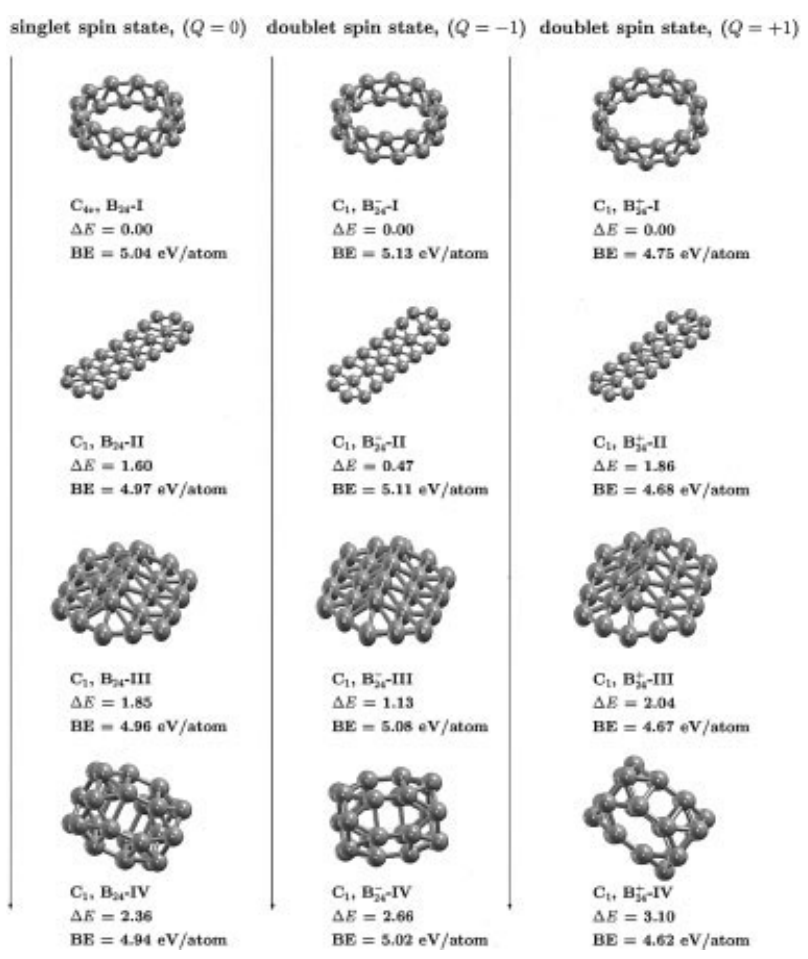

FIGURE 2. Energy differences ( $\Delta E$ in eV), binding energy (eV/atom), and symmetry for some of the low-lying isomers of neutral, anionic and cationic $B_{24}$ clusters.

$\AA$ Abtained by the DFT-planewave calculations [19] for the double-ring configuration.

The next low-lying isomer is an elongated quasiplanar configuration $\left(\mathrm{B}_{24}-\mathrm{II}\right)$, which is $1.60 \mathrm{eV}$ above the double-ring isomer. After the optimization, $C_{2 h}$ configuration leads to $C_{1}$ symmetry. It is worth

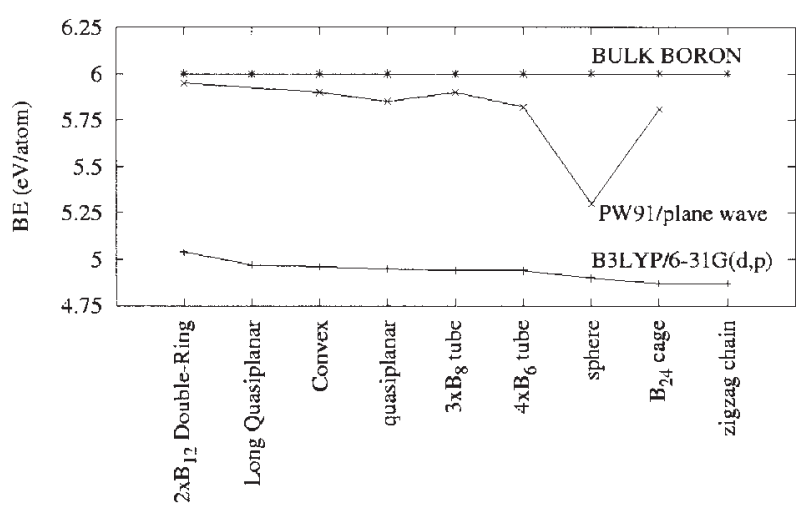

FIGURE 3. Binding energy (BE) per atom (in eV) of several isomers taken from this work, and from the DFT-plane wave calculation [14] in comparison with boron bulk binding energy [23] are shown here. 
mentioning that a competition between the doublering and elongated quasi-planar structures in becoming the ground-state configuration can be understood on the basis of curvature strain (favoring quasi-planar structure) and elimination of dangling bonds (favoring double-ring structure) [22]. Two possible tubular structures, namely $3 \times \mathrm{B}_{8}$ and $4 \times$ $\mathrm{B}_{6}$ were also considered here. Total energy calculations without the symmetry-constraint optimization for $D_{4 h}$ configuration of $3 \times \mathrm{B}_{8}$ tubular structure yields a "square-shape" tube with $C_{1}$ symmetry. It is also the case with $D_{6 d}$ configuration of $4 \times \mathrm{B}_{6}$ tubular structure $\left(\mathrm{B}_{24}-\mathrm{IV}\right)$, which goes to $C_{1}$ symmetry after the geometry optimization. Here, the calculated diameter is 3.2 and $4.0 \AA$ at the open end and at the center, respectively. It is to be noted here that both $D_{4 h}$ configuration of $3 \times \mathrm{B}_{8}$ and $D_{6 d}$ configuration of $4 \times \mathrm{B}_{6}$ structures considered in the previous studies $[18,19]$ are, in fact, saddle points on the potential energy surface with imaginary frequencies in their vibrational spectra.

The 3D isomeric configurations, such as a $D_{6 d}$ cage or a spherical configuration are found to be more than $3 \mathrm{eV}$ higher than the calculated ground state of $B_{24}$. It therefore suggests that the reported [24-26] stable spherical cage structures of $C_{24}, C_{32}$, and $C_{36}$ are not likely to be preferred configurations for the large boron clusters.

\subsubsection{Anionic and Cationic $B_{24}$}

All the anionic isomeric configurations of $\mathrm{B}_{24}$ are found to be in doublet spin state, and are more stable in energy than their respective neutral species. The ordering of these configurations in terms of total energy is almost similar to what was calculated for the neutral $B_{24}$. Addition of an electron tends to induce a larger distance between the rings during the structural optimization. The lowest energy configuration is the double-ring $\left(\mathrm{B}_{24}^{-1}-\mathrm{I}\right)$ structure in $C_{1}$ symmetry. We note that the symmetric $C_{4 v}$ double-ring structure is only $0.06 \mathrm{eV}$ higher in energy, making it to be nearly degenerate with the $C_{1}$ double-ring configuration. The next isomer is the elongated quasi-planar configuration which is about $0.47 \mathrm{eV}$ higher in energy with respect to the $C_{1}$ double-ring isomer.

As the case with the anionic $\mathrm{B}_{24}$, the removal of an electron does not alter the ordering of cationic $\mathrm{B}_{24}$ isomers, as shown in Figure 2. The lowest energy configuration is still the $C_{1}$ double-ring structure $\left(\mathrm{B}_{24}^{+}-\mathrm{I}\right)$ with a diameter of about $6.20 \AA$, around

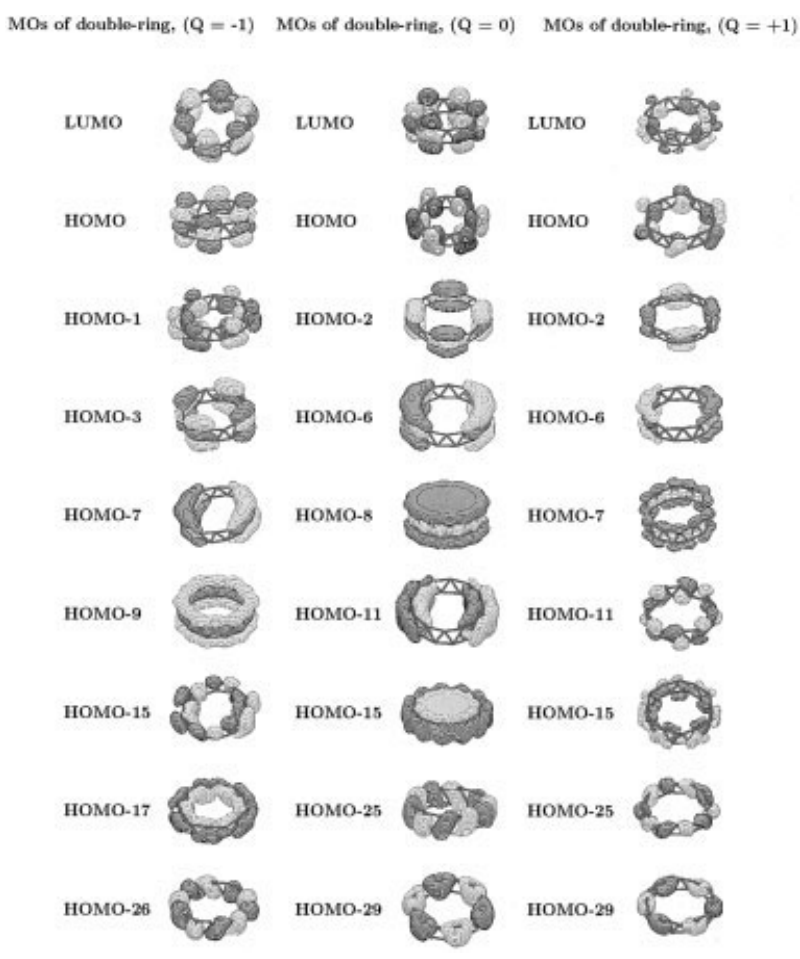

FIGURE 4. The selected molecular orbitals (MOs) of neutral, anionic, and cationic double-ring $\mathrm{B}_{24}$ configuration.

$0.06 \mathrm{eV}$ more stable than cationic $C_{4 v}$ double-ring structure.

\subsection{BONDING AND STABILITY}

In boron clusters, the chemical bonding is dominated by the electron-deficient character in a sense that more atomic orbitals are available for the bonding than electrons associated with atoms. Thus multi-center bonds such as the three-center triangular B-B-B units are expected to become a key bonding feature in boron compounds to accommodate their electron deficiency $[11,14]$.

In Figure 4, we present the analysis of the molecular orbitals (MOs) for the ground state of $B_{24}$, $\mathrm{B}_{24}^{-}$, and $\mathrm{B}_{24}^{+}$. This analysis reveals the interesting features of delocalized $\pi, \sigma$, and multicentered $\sigma$ bonds between the boron atoms in these clusters. Instead of giving exhaustive description of these MOs, we will only extract general features of the bonding orbitals using the notation HOMO- $n$ to represent the $(60-n)^{\mathrm{th}}$ occupied molecular orbital in the neutral cluster. 
As shown in Figure 4, the highest occupied molecular orbital (HOMO) shows the $\pi$ bond between the atoms of each ring, while the lowest unoccupied molecular orbital (LUMO) shows a lateral p-p overlap between the atoms of both rings. The HOMO and (HOMO-1) are doubly degenerate giving rise to an effective delocalization. On the other hand, (HOMO-2) and (HOMO-3) orbitals show localized $\pi$-bonding in $\mathrm{B}_{1}$ state. The (HOMO-6) and (HOMO-7) degenerate orbital show a delocalized $\pi$-bonding at the sides in E state. The full-delocalization of benzene-like $\pi$ bonds between the two staggered rings is achieved in $\mathrm{A}_{1}$ state of the (HOMO-8) orbital. Besides being facilitated by the delocalized $\pi$ bonds perpendicular to the plane of the double-ring structure, the benzene-like delocalized $\pi$ bonding in plane is shown in the doubledegenerate (HOMO-11) and (HOMO-12) orbitals. A strong multicentered $\sigma$ bonds is shown in the (HOMO-25) and (HOMO-29) orbitals in E state which are formed by the hybridization of $\mathrm{s}$ - and p-type bonds, also seen in the neutral $B_{12}$ [20].

The anionic double-ring configuration shows the similar features to those seen in the neutral case (Fig. 4). The HOMO is, in fact, the LUMO of the neutral cluster. Addition of an extra electron to LUMO of the neutral $B_{24}$ shifts the anionic HOMO to the lower energy relative to the energy of the neutral HOMO. It has also resulted in a shifting of the localized $\pi$ bonds and the "benzene-like" delocalized $\pi$ bonds to (HOMO-3), (HOMO-7), and (HOMO-9) orbitals, respectively, in the anionic $\mathrm{B}_{24}$. Although the cationic double-ring configuration is found to have a similar features in their MOs, magnitude of the electron delocalization is relatively less than that of either neutral or anionic configurations. For example, the electron density plots of the (HOMO-7) and (HOMO-15) $\alpha$-orbitals appear to be less dense and less delocalized. Furthermore, the benzene-like delocalized $\pi$ bonding is not seen in MOs of the cationic $\mathrm{B}_{24}$. We believe that absence of these features in MOs of the cationic $\mathrm{B}_{24}$ may explain why the cationic double-ring configuration is energetically less stable than its corresponding neutral and anionic configurations.

Analysis of MOs of the other isomers of $\mathrm{B}_{24}$ also reveals that the delocalized $\pi$ and multicentered $\sigma$ bonds between boron atoms of the quasi-planar and convex isomers are "fragmented" into different parts of the cluster configurations. On the other hand, the dominant features in MOs of 3D isomers are mainly associated with the localized orbitals. We may therefore conclude that the presence of delocalized $\pi$ and multicentered $\sigma$ bonds play an important role in stabilizing the double-ring configuration over the 2D quasi-planar and other 3D configurations for the neutral and ionized $\mathrm{B}_{24}$ clusters.

\subsection{ELECTRON AFFINITY, IONIZATION POTENTIAL, AND HOMO-LUMO GAP}

Both adiabatic and vertical values of the electron affinity and ionization potential for some of the low-lying isomers of $\mathrm{B}_{24}$ are reported here. The values of electron affinity (EA) and ionization potential (IP) are obtained as follows: $E A=E_{(q=0)}$ $-E_{(q=-1)}$, and IP $=E_{(q=+1)}-E_{(q=0)}$, where $E$ is the total energy of the cluster and $q$ is the charge on the cluster. In this way, the vertical electron affinity (VEA) or vertical detachment energy (VDE) [27] is defined as the energy difference between the anionic and neutral clusters with both at the optimized geometry of the anionic cluster. Adiabatic electron affinity (AEA) is defined as the energy difference between the anionic and neutral clusters at their own respective optimized geometries.

To calculate VDE, we use the $6-311+\mathrm{G}(\mathrm{d})$ basis set for the equilibrium structure obtained using the $6-31 G(d, p)$ basis set with an aim to obtain a more accurate value of energies, knowing that the calculated equilibrium structure of double-ring $\left(\mathrm{B}_{24}-\mathrm{I}\right)$ remains nearly the same for both basis sets. Employing a similar approach, we also calculate the vertical attachment energy (VAE) which defined as the energy difference between the neutral and anionic clusters with both at the optimized geometry of the neutral cluster. The calculated VAE and VDE values come out to be 2.67 and $2.81 \mathrm{eV}$, respectively. Thus, if the nuclear configuration of the negative ion does not drastically differ from that of the uncharged parent species, the VAE and VDE will provide lower and upper bounds, respectively [27], for the electron affinity of the double-ring $B_{24}$.

The vertical ionization potential (VIP) is defined as the energy difference between the cationic and neutral clusters with both at the optimized geometry of the neutral cluster; the adiabatic ionization potential (AIP) is defined as the energy difference between the cationic and neutral clusters at their own respective optimized geometries. Based on the 6-31G $(d, p)$ basis set, the VIP and AIP values of the lowest energy configuration (double-ring, $\mathrm{B}_{24}-\mathrm{I}$ ) is $6.88 \mathrm{eV}$ and $6.80 \mathrm{eV}$, respectively. For elongated quasi-planar structure $\left(\mathrm{B}_{24}-\mathrm{II}\right)$, the VIP and AIP values are 7.19 and $7.06 \mathrm{eV}$, respectively. And for convex structure $\left(\mathrm{B}_{24}-\mathrm{III}\right)$, its VIP and AIP is 7.12 


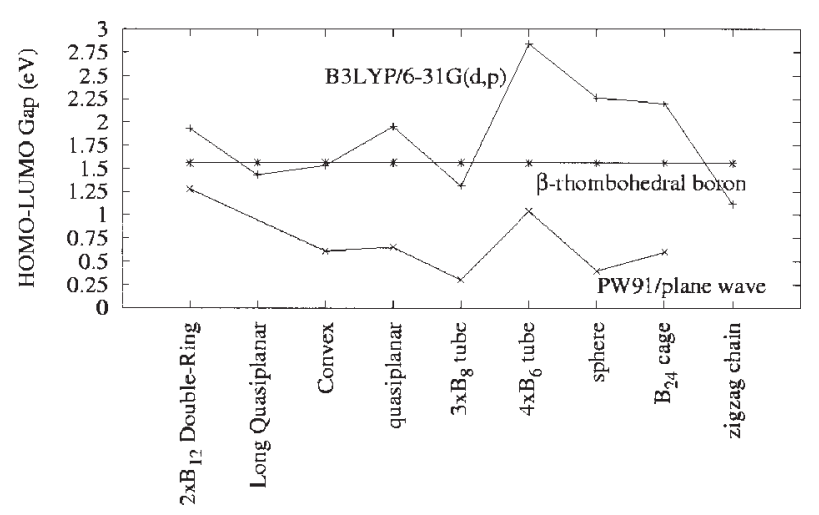

FIGURE 5. Energy separation (in eV) of the HOMO and LUMO of several isomers taken from this work, and from the DFT-plane-wave calculations [19], in comparison with the band gap of the $\beta$-rhombohedral boron [23] are shown here.

and $6.98 \mathrm{eV}$. A comparison of VIP and AIP values of these cationic isomers with those of small cationic boron clusters, $\mathrm{B}_{n}^{+}(n=2-14)[7,20]$ indicates that the IPs of $\mathrm{B}_{24}$ is showing a decreasing trend towards the bulk boron work function [28] of $4.6 \mathrm{eV}$. By applying the concept of quantification of chemical hardness [29] of a particular system as $\eta \approx$ (IP EA) $/ 2$ where IP and EA are the first vertical ionization energy and electron affinity of the chemical species, the double-ring $B_{24}$ is found to be chemically inert by having the highest value of $\eta$.

The calculated HOMO-LUMO gaps of the neutral isomers of $\mathrm{B}_{24}$ are shown in Figure 5 and are slightly higher than the bulk value of $1.56 \mathrm{eV}$ of the $\beta$-rhombohedral boron [23]. The calculated HOMOLUMO gap of the neutral double-ring structure $\left(\mathrm{B}_{24}-\mathrm{I}\right)$ is $1.93 \mathrm{eV}$. For anionic and cationic doublering isomer, the HOMO-LUMO gaps are $0.62 \mathrm{eV}$ and $1.13 \mathrm{eV}$, respectively. It is well known that the hybrid functional form used in the present study is more reliable in predicting the band gap in semiconducting and ionic materials $[30,31]$.

\subsection{VIBRATIONAL PROPERTIES}

\subsubsection{Vibrational Spectrum of $B_{24}, B_{24}^{-}$, and $\mathrm{B}_{24}^{+}$}

Figure 6 shows the distribution of the calculated vibrational frequencies of neutral, anionic, and cationic $B_{24}$ that lie in the range of $30-1500 \mathrm{~cm}^{-1}$. In general, addition or removal of an electron only shifts the values of the frequencies, and does not induce any significant changes in their spectra. It is noteworthy that the calculated spectra of the double-ring and $\left(4 \times \mathrm{B}_{6}\right)$ tubular structures are almost similar. The tubular structure can be considered as a segment of the boron nanotube, and has vibrational properties similar to those of carbon and boron nitride nanotubes [32, 33]. For example, the frequency region below $500 \mathrm{~cm}^{-1}$ in the $\left(4 \times \mathrm{B}_{6}\right)$ tubular structure are associated with different "radial buckling modes," such as $\mathrm{E}, \mathrm{B}_{1}, \mathrm{~B}_{2}, \mathrm{~A}_{1}$, and $\mathrm{A}_{2}$ modes, as the case with carbon nanotubes [33].

In the neutral and ionized double-ring $\mathrm{B}_{24}$, the low-frequency modes $\left(\nu \leq 500 \mathrm{~cm}^{-1}\right)$ correspond to vibrations in the "radial modes." In the neutral case, the lowest radial mode, $\nu_{\text {lowest }}$ is at $77 \mathrm{~cm}^{-1}$ which is associated with two nearly degenerated $B_{1}$ and $\mathrm{B}_{2}$ modes. The "radial breathing modes" at 381 and $389 \mathrm{~cm}^{-1}$ are $\mathrm{A}_{1}$ modes. It is to be noted here that the radial modes in the region ranging from 200 to $500 \mathrm{~cm}^{-1}$ have recently been identified in the boron nanotubes by Raman experiments [6].

The "longitudinal modes" along the ring (tubular) axis are found to be in the region of $600-900$ $\mathrm{cm}^{-1}$, while the "tangential modes" due to stretching of the stronger $\mathrm{B}-\mathrm{B}$ covalent bonds along the ring circumference are associated with frequencies above $900 \mathrm{~cm}^{-1}$. The highest frequency of the tangential mode, $\nu_{\text {highest }}$ is $1325 \mathrm{~cm}^{-1}$ in neutral $B_{24}$ double-ring. Addition of an electron to the neutral double-ring $\mathrm{B}_{24}$ reduces both $\nu_{\text {lowest }}$ and $\nu_{\text {highest }}$ to 68 and $1323 \mathrm{~cm}^{-1}$ respectively. On the other hand, the removal of an electron reduces $\nu_{\text {lowest }}$ to 41 $\mathrm{cm}^{-1}$, while increasing $\nu_{\text {highest }}$ to $1259 \mathrm{~cm}^{-1}$.

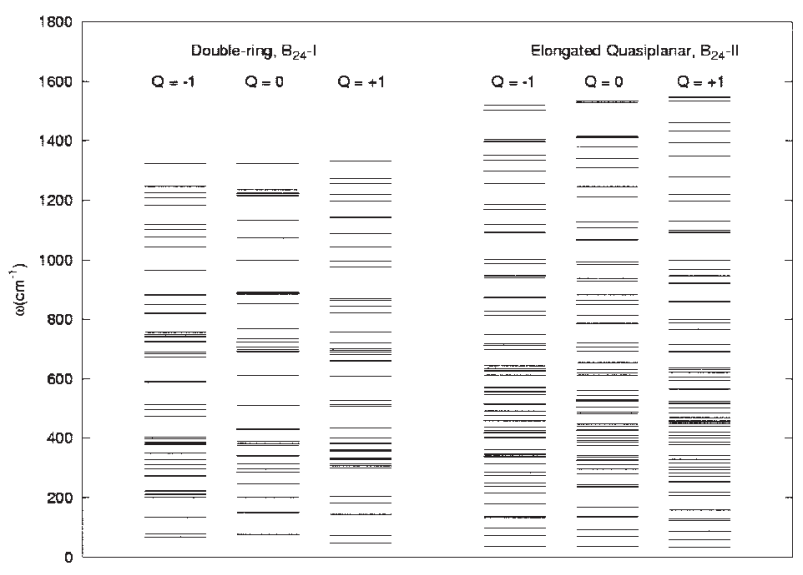

FIGURE 6. The calculated vibrational frequencies of the double-ring $\left(\mathrm{B}_{24}-\mathrm{l}\right)$ and elongated quasi-planar $\left(\mathrm{B}_{24^{-}}\right.$ II) structures in the neutral, positive, and negative charge states. 
The frequency spectrum of the $\left(4 \times \mathrm{B}_{6}\right)$ tubular structure shows the same unique features in the vibrational modes that were seen in the double-ring configuration. The low-frequency region below 500 $\mathrm{cm}^{-1}$ is dominated by the "radial buckling modes," the lowest radial mode being at $139 \mathrm{~cm}^{-1}$. The radial breathing mode associated with the open ends is at $207 \mathrm{~cm}^{-1}$. On the other hand, the breathing modes associated with the center of the tube are at 292 and $299 \mathrm{~cm}^{-1}$. The tangential modes are dominant in the frequency region of $500-900 \mathrm{~cm}^{-1}$, and the longitudinal modes via vibrations along the tubular axis are at about $1248 \mathrm{~cm}^{-1}$. Because both double-ring and $\left(4 \times \mathrm{B}_{6}\right)$ tubular structures exhibit the vibrational modes, which are known to be typical characteristic vibrational modes reported in $\mathrm{C}$ and $\mathrm{BN}$ nanotubes, these tubular structures can be considered as a basic building block of boron nanotubes. For example, $\left(4 \times \mathrm{B}_{6}\right)$ structure can be considered as a segment of a boron nanotube with a radius of $0.33 \mathrm{~nm}$.

Compared to the double-ring and other tubular structures of $\mathrm{B}_{24}$, the elongated quasi-planar structure $\left(\mathrm{B}_{24}-\mathrm{II}\right)$ is associated with a distinctively different distribution of frequencies, as shown in Figure 6 . The lowest frequency of the neutral isomer, $\nu_{\text {lowest }}$ is $36 \mathrm{~cm}^{-1}$. The high-frequency region is dominated by the symmetric and asymmetric stretching modes. The highest vibrational frequency, $\nu_{\text {highest }}$ is at $1535 \mathrm{~cm}^{-1}$ associated with the asymmetric stretching of atoms along the edge of the plane.

\subsubsection{IR Spectra}

The calculated (Lorentzian-broadened) infrared (IR) spectra of some of the isomeric configurations of neutral and ionized $\mathrm{B}_{24}$ clusters are shown in Figure 7 . We note that the prediction of infrared intensities can be accomplished by using the mixed second derivatives of energy with respect to geometric motion and an external electric field by permitting estimation of changes in the dipole moment as a function of the vibrations. Therefore the intensity $(\mathrm{km} / \mathrm{mol})$ of an infrared absorption band is proportional to the square of change in molecular electric dipole moment $\mu$ in the normal coordinates (i.e., $I_{i}=$ const $\times\left(\partial \mu / \partial Q_{i}\right)^{2}$ ).

The double-ring $\mathrm{B}_{24}$ configuration shows the IRactive E modes at 512 and $852 \mathrm{~cm}^{-1}$ arising from its $C_{4 v}$ symmetry. The peak at $512 \mathrm{~cm}^{-1}$ is due to the tangential mode, whereas the strongest peak at 852 $\mathrm{cm}^{-1}$ corresponds to the longitudinal mode. In the
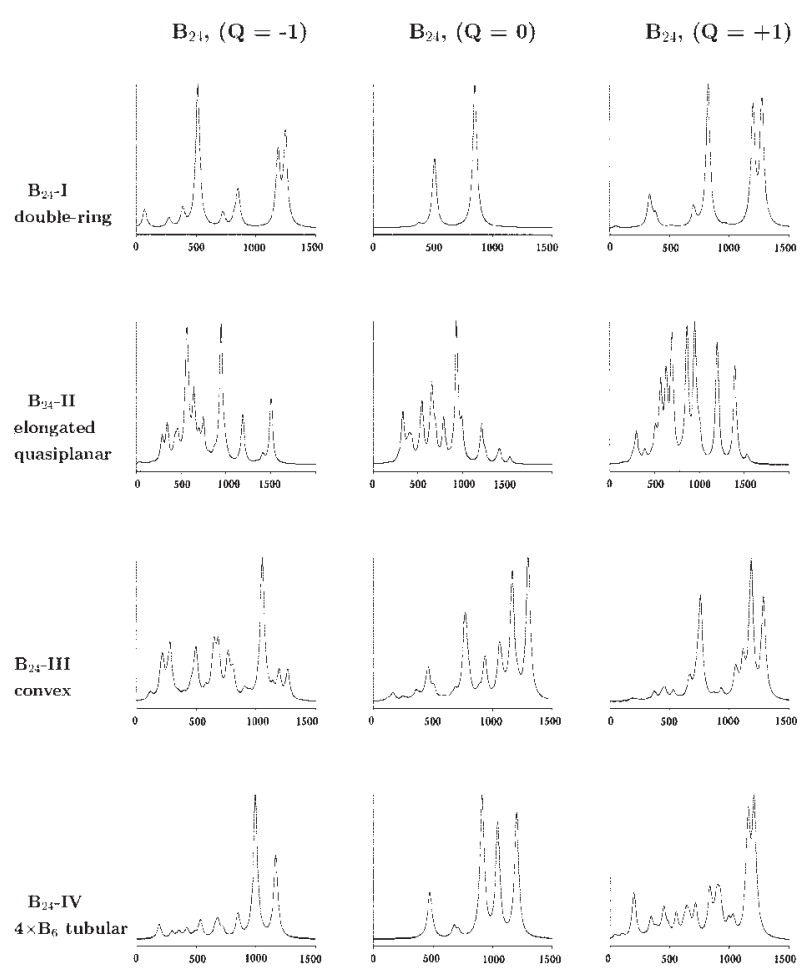

FIGURE 7. Infrared (IR) spectra for different isomers of the neutral, anionic, and cationic $\mathrm{B}_{24}$ clusters.

ionized $\mathrm{B}_{24}$, distribution of the IR intensities is altered due to dissimilar atomic charges. The noticeable IR peaks are at 515 and $1184 \mathrm{~cm}^{-1}$ in $\mathrm{B}_{24}^{-}$, while they are at 822,1198 , and $1273 \mathrm{~cm}^{-1}$ in $B_{24}^{+}$.

In the $\left(4 \times \mathrm{B}_{6}\right)$ tubular structure, the IR peaks are more evenly distributed than those in the doublering structure. In the low-frequency region, the IR peak at $469 \mathrm{~cm}^{-1}$ is associated with the radial buckling mode. There are two nearly degenerate tangential modes at 911 and $913 \mathrm{~cm}^{-1}$. The other two distinctive IR peaks located at 1042 and $1201 \mathrm{~cm}^{-1}$ are associated with the longitudinal modes along the tubular axis. The neutral quasi-planar and convex $\left(\mathrm{B}_{24}-\mathrm{II}\right.$ and $\mathrm{B}_{24}$-III) structures, in general, have relatively more widely distributed IR peaks due to their 2D extended planar configurations.

\subsubsection{Polarizability}

Table I collects the calculated dipole moments of the neutral and ionized isomers of $B_{24}$ whose values reflect a close relationship with their structural symmetry. Ionization-induced changes in the dipole moments become noticeable only for some of the isomers. For example, the added electron to the 
TABLE I

Dipole moment ( $\mu_{i}$ in Debye), diagonal components of static polarizability tensor ( $\alpha_{j j}$ in a.u.), and average static polarizability ( $\alpha_{\mathrm{av}}$ in a.u.) for the neutral, anionic, and cationic isomers of $B_{24}$.

\begin{tabular}{|c|c|c|c|c|c|c|c|c|c|c|c|c|}
\hline \multirow[b]{2}{*}{ Properties } & \multicolumn{4}{|c|}{$\mathrm{B}_{24}, \mathrm{Q}=0$} & \multicolumn{4}{|c|}{$\mathrm{B}_{24}, \mathrm{Q}=-1$} & \multicolumn{4}{|c|}{$\mathrm{B}_{24}, \mathrm{Q}=+1$} \\
\hline & $\mathrm{B}_{24}-\mathrm{I}$ & $\mathrm{B}_{24}-\mathrm{II}$ & $\mathrm{B}_{24}-\mathrm{III}$ & $\mathrm{B}_{24}-\mathrm{VI}$ & $\mathrm{B}_{24}^{-}-\mathrm{I}$ & $\mathrm{B}_{24}^{-}-\mathrm{II}$ & $\mathrm{B}_{24}^{-}-\mathrm{III}$ & $\mathrm{B}_{24}^{-}-\mathrm{VI}$ & $\mathrm{B}_{24}^{+}-\mathrm{I}$ & $\mathrm{B}_{24}^{+}-\mathrm{II}$ & $\mathrm{B}_{24}^{+}-\mathrm{III}$ & $\overline{\mathrm{B}_{24}^{+}-\mathrm{VI}}$ \\
\hline$\mu_{x}$ & 0.0000 & -0.0040 & 0.0000 & -0.0002 & -0.0047 & 0.0080 & 0.0046 & -0.0017 & 0.0039 & -0.0075 & -0.0093 & -0.0008 \\
\hline$\mu_{y}$ & 0.0000 & -0.0006 & 0.0000 & -0.0009 & -0.0054 & 0.0072 & 0.0000 & -0.0014 & 0.0001 & -0.0022 & 0.0004 & -0.1420 \\
\hline$\mu_{z}$ & 0.0001 & 0.0077 & 0.4982 & -0.0003 & 0.0078 & 0.0064 & 1.1546 & -0.0017 & -0.0199 & 0.0010 & 0.4701 & 0.0161 \\
\hline$\mu_{\text {Tot }}$ & 0.0001 & 0.0087 & 0.4982 & 0.0009 & 0.0106 & 0.0125 & 1.1546 & 0.0028 & 0.0203 & 0.0079 & 0.4702 & 0.1429 \\
\hline$\alpha_{x x}$ & 413.43 & 934.89 & 479.67 & 280.64 & 420.35 & 984.37 & 549.22 & 303.66 & 407.09 & 943.75 & 471.21 & 291.77 \\
\hline$\alpha_{y y}$ & 413.43 & 292.67 & 386.05 & 230.46 & 420.25 & 303.73 & 426.77 & 249.40 & 406.80 & 291.40 & 371.59 & 225.03 \\
\hline$\alpha_{z z}$ & 199.62 & 115.94 & 124.68 & 230.37 & 206.94 & 114.77 & 128.40 & 249.33 & 194.97 & 112.81 & 123.13 & 225.24 \\
\hline$\alpha_{\text {avg }}$ & 342.16 & 447.83 & 330.13 & 247.16 & 349.18 & 467.62 & 368.13 & 267.46 & 336.29 & 449.32 & 321.98 & 247.35 \\
\hline
\end{tabular}

neutral tubular structure appears to be shared by all the atoms. In the cationic tubular structure, however, the ionized electron appears to come from only few atoms.

Instead of discussing the static dipole polarizability in terms of the parallel $\left(\alpha_{x x}\right)$, transverse $\left(\alpha_{y y}\right)$, and perpendicular $\left(\alpha_{z z}\right)$ components, we have considered the directional averaged static polarizability, $\alpha_{\text {avg }}=\left(\alpha_{x x}+\alpha_{y y}+\alpha_{z z}\right) / 3$ to extract a general trend. Accordingly, $\alpha_{\text {avg }}$ of the $\mathrm{B}_{24}$ double-ring configuration shows a large increase relative to the reported value of 146.79 for the convex $B_{12}$ structure [20]. It is consistent with the trend in which $\alpha_{\text {avg }}$ is expected to increase with the size of cluster $[20,24,35]$. The double-ring $\mathrm{B}_{24}$ is found to be significantly less polarizable than the elongated quasi-planar $B_{24}$, suggesting that delocalization of charge distribution plays an important role in reducing the static dipole polarizability. In the elongated quasi-planar structure, the $\pi$-electron population is expected to be localized along the chain direction. The addition or removal of an electron does not introduce any significant changes in the $\alpha_{\text {avg }}$ as shown in Table I.

\section{Summary}

The equilibrium structures, energetics, chemical bonding, electronic and vibrational properties, and static dipole polarizability of the neutral and ionized $\mathrm{B}_{24}$ clusters is reported here. Calculations based on DFT find ionization-induced changes in the structural parameters to be small. The ordering of the isomeric configuration does not change upon addition or removal of an electron. The staggered double-ring configuration which facilitates $\mathrm{sp}^{2}$ hybridization in the cluster is the lowest energy configuration. The presence of the typical characteristic vibrational modes which are associated with $C$ and $\mathrm{BN}$ nanotubes in the vibrational frequency spectrum of the double-ring and $\left(4 \times \mathrm{B}_{6}\right)$ tubular structures suggest that these structures appear to be the building blocks of boron nanotubes.

\section{ACKNOWLEDGMENT}

We gratefully acknowledge CSERC, MTU, for access to their computing facility. We acknowledge helpful discussions with S. Chacko, M.D.D. gratefully acknowledges for providing the local hospitality at Michigan Technological University.

\section{References}

1. Kroto, H. W.; Heath, J. R.; O'Brien, S. C.; Curl, R. F.; Smalley, R. E. Nature 1985, 318, 162.

2. Ijima, S. Nature (London) 1991, 354, 56.

3. Quasicrystals: The State of the Art; DiVincenzo, D. P.; Steinhardt, P. J., Eds.; World Scientific: Singapore, 1991.

4. Cao, L. M.; Hahn, K.; Scheu, C.; Ruhle, M.; Wang, Y. Q.; Zhang, Z.; Gao, C. X.; Li, Y. C.; Zhang, X. Y.; He, M.; Sun, L. L.; Wang, W. K. Appl Phys Lett 2002, 80, 4226.

5. Xu, T. T.; Zheng, J. G.; Wu, N.; Nicholls, A. W.; Roth, J. R.; Dikin, D. A.; Ruoff, R. S. Nanoletters 2004, 4, 963.

6. Ciuparu, D.; Klie, R. F.; Zhu, Y.; Pfefferle, L. J Phys Chem B 2004, 108, 3967

7. Boustani, I. Int J Quantum Chem 1994, 52, 1081.

8. Boustani, I. Phys Rev B 1997, 55, 16426.

9. Niu, J.; Rao, B. K.; Jena, P. J. J Chem Phys 1997, 107, 132.

10. Alexandrova, A. N.; Boldyrev, A. I.; Zhai, H. J.; Wang, L. S.; Steiner, E.; Fowler, P. W. J Phys Chem A 2003, 107, 1359. 


\section{LAU ET AL.}

11. Zhai, H. J.; Kiran, B.; Li, J.; Wang, L. S. Nature Mater 2003, 2, 827.

12. Alexandrova, A. N.; Boldyrev, A. I.; Zhai, H. J.; Wang, L. S. J Phys Chem A 2004, 108, 3509.

13. Zhai, H. J.; Alexandrova, A. N.; Birch, K. A.; Boldyrev, A. I.; Wang, L. S. Angew Chem Int Ed 2003, 42, 6004.

14. The Chemistry of Boron and Its Compounds; Muetterties, E. L., Ed.; John Wiley: New York, 1967.

15. Bylander, D. M.; Kleinman, L.; Lee, S. Phys Rev B 1990, 42, 1394.

16. Perkins, C.; Trenary, M.; Tanaka, T. Phys Rev B 1998, 58, 9980.

17. Vast, N.; Baroni, S.; Zerah, G.; Besson, J. M.; Polian, A.; Grimsditch, M.; Chervin, J. C. Phys Rev Lett 1997, 78, 693.

18. Boustani, I.; Quandt, A. Europhys Lett 1997, 39, 527.

19. Chacko, S.; Kanhere, D. G.; Boustani, I. Phys Rev B 2003, 68, 035414.

20. Lau, K. C.; Deshpande, M.; Pandey, R. Int J Quantum Chem 2004, in press.

21. Frisch, M.; Trucks, G. W.; Schlegel, H. B.; Scuseria, G. E.; Robb, M. A.; Cheeseman, J. R.; Zakrzewski, V. G.; Montgomery, J. A.; Stratmann, R. E.; Burant, J. C.; Dapprich, S.; Millam, J. M.; Daniels, A. D.; Kudin, K. N.; Strain, M. C.; Farkas, O.; Tomasi, J.; Barone, V.; Cossi, M.; Cammi, R.; Mennucci, B.; Pomelli, C.; Adamo, C.; Clifford, S.; Ochterski, J.; Petersson, G. A.; Ayala, P. Y.; Cui, Q.; Morokuma, K.; Malick, D. K.; Rabuck, A. D.; Raghavachari, K.; Foresman, J. B.; Cioslowski, J.; Ortiz, J. V.; Stefanov, B. B.; Liu, G.; Liashenko, A.; Piskorz, P.; Komaromi, I.; Gomperts, R.; Martin, R. L.; Fox, D. J.; Keith, T.; Al-Laham, M. A.; Peng, C. Y.; Nanayakkara, A.;
Gonzalez, C.; Challacombe, M.; Gill, P. M. W.; Johnson, B. G.; Chen, W.; Wong, M. W.; Andres, J. L.; Head-Gordon, M.; Replogle, E. S.; Pople, J. A. Gaussian 98 Gaussian, Inc.: Pittsburgh, PA, 1998.

22. Boustani, I.; Rubio, A.; Alonso, J. A. Chem Phys Lett 1999, 311, 21.

23. Lide, D. R. CRC Handbook of Chemistry and Physics; CRC Press: Boca Raton, FL, 1995.

24. Jones, R. O.; Seifert, G. Phys Rev Lett 1997, 79, 443.

25. Jones, R. O. J Chem Phys 1999, 110, 5189.

26. Grossman, J. C.; Colvin, M. E.; Tran, N. L.; Louie, S. G.; Cohen, M. L. Chem Phys Lett 2002, 356, 247.

27. Rienstra-Kiracofe, J. C.; Tschumper, G. S.; Schaefer, H. F. III. Chem Rev 2002, 102, 231.

28. Hanley, L.; Whitten, J. L.; Anderson, S. L. J Phys Chem 1988, 92, 5803.

29. Pearson, R. G. Chemical Hardness; Wiley-VCH Verlag: Weinheim, Germany, 1997.

30. Jiang, H.; Orlando, R.; Blanco, M. A.; Pandey, R. J Phys Condens Matter 2004, 16, 3081.

31. Harrison, N. M.; Wander, A.; Muscat, J. Chem Phys Lett 2001, 342, 397.

32. Ciuparu, D.; Chen, Y.; Lim, S.; Haller, G. L.; Pfefferle, L. J. J Phys Chem B 2004, 108, 503.

33. Wirtz, L.; Rubio, A. IEEE Trans Nanotechnol 2003, 2, 341.

34. Reis, H.; Papadopoulos, M. G.; Boustani, I. Int J Quantum Chem 2000, 78, 131.

35. Abdurahman, A.; Shukla, A.; Seifert, G. Phys Rev B 2002, 66, 155423. 